\title{
Estados emocionais de casais submetidos à fertilização in vitro'
}

\author{
Emotional states of couples undergoing \\ in vitro fertilization
}

\author{
Helena Maria Loureiro MONTAGNINI ${ }^{5}$ \\ Sérgio Luís BLAY² \\ Neil Ferreira NOVO ${ }^{3}$ \\ Vilmon de FREITAS ${ }^{4}$ \\ Agnaldo Pereira CEDENHO
}

\begin{abstract}
Resumo
A infertilidade interrompe um projeto de vida pessoal e do casal, produzindo sofrimento psíquico. O desenvolvimento das técnicas de reprodução assistida possibilitou a solução para casais que anteriormente não teriam alternativas de tratamento. Assim, a fertilização in vitro constitui uma nova fonte de esperança para ter um filho, mas, ao mesmo tempo, pode ser acompanhada de muitas dificuldades. O objetivo deste estudo foi comparar os estados emocionais de homens e mulheres submetidos à fertilização in vitro e verificar a relação entre estado emocional das mulheres e ocorrência de gravidez. Foram avaliados vinte casais submetidos ao primeiro ciclo de fertilização in vitro, entre o $5^{\circ}$ e $010^{\circ}$ dia após a transferência dos pré-embriões. A avaliação foi realizada por meio de testes psicológicos e entrevistas. As mulheres apresentaram níveis mais altos de ansiedade e depressão que os homens. A autoestima delas mostrou-se mais baixa. Um quarto das mulheres que apresentaram sintomas psicoemocionais não engravidou, porém esta relação não foi significativa. Concluiu-se que as mulheres apresentaram mais ansiedade e sintomas depressivos que os homens e autoestima mais baixa. O grupo de mulheres com sintomas psicoemocionais apresentou tendência a não engravidar.
\end{abstract}

Unitermos: Ansiedade. Auto-imagem. Depressão. Infertilidade.

\begin{abstract}
Infertility intrudes into the plans in one's personal life and the life of a couple, and causes mental suffering. In vitro fertilization is a new technique in reproductive technology and offers fresh hope of having children, but at the same time, it is considered to be a stressful experience. The aim of this study is to compare the emotional state of men and women undergoing in vitro fertilization and identify a possible relationship
\end{abstract}

UVV

1 Artigo elaborado a partir de dissertação de H.M.L. MONTAGNINI, intitulada "Aspectos emocionais de casais submetidos à fertilização in vitro". Universidade Federal de São Paulo. Apoio: Coordenação de Aperfeiçoamento de Pessoal de Nível Superior.

2 Universidade Federal de São Paulo, Departamento de Psiquiatria. São Paulo, SP, Brasil.

3 Faculdade de Medicina de Santo Amaro, Disciplina de Saúde Pública. Santo Amaro, SP, Brasil.

4 Universidade Federal de São Paulo, Departamento de Ginecologia. São Paulo, SP, Brasil.

5 Universidade Federal de São Paulo, Departamento de Cirurgia, Serviço de Reprodução Humana. R. Botucatu, 725, V. Clementina, 04023-900, São Paulo, SP, Brasil. Correspondência para/Correspondence to: H.M.L. MONTAGNINI. E-mail: <hmmontag.uol.com.br>. 
between women's emotional state and a successful outcome. Twenty couples in their first cycle of in vitro fertilization were included in this study. Men and women were submitted individually to psychological interviews and tests between 5 and 10 days after the transfer of pre-embryos. Women presented higher levels of anxiety and depression than men. Their self-esteem proved to be lower. Those women (25\%) that presented psycho-emotional symptoms, did not get pregnant, however this relationship was not significant. Based on these findings, we conclude that women presented greater anxiety and symptoms of depression than the men and had lower self-esteem. The group of women with psycho-emotional symptoms displayed a tendency not to get pregnant.

Uniterms: Anxiety. Self-concept. Depression. Infertility.

A infertilidade interrompe um projeto de vida pessoal e do casal, produzindo sofrimento psíquico. Para alguns, ter um filho é o principal objetivo de vida e, nesses casos, o sofrimento decorrente da infertilidade é maior (Collins, Freeman, Boxer \& Tureck, 1992). Homens e mulheres têm maneiras diferentes de experienciar e lidar com as dificuldades decorrentes da infertilidade e seu tratamento (Wright et al.,1 1991).

A Fertilização In Vitro (FIV) constitui uma nova esperança para ter um filho, mas, ao mesmo tempo, pode ser acompanhada de muitas dificuldades e desapontamentos.

Ao iniciar o primeiro procedimento de FIV, os casais mostram-se muito otimistas e superestimam suas chances de sucesso. Apresentam níveis normais de ansiedade, depressão e autoestima, não se evidenciando diferenças significativas entre homens e mulheres (Hearn, Yuzpe, Brown \& Casper, 1987). Edelmann, Connolly e Bartlett (1994) consideraram a existência de uma seleção natural, em que somente aqueles que têm condições de lidar com as dificuldades do tratamento chegam a procurá-lo. Nessa mesma linha, Beaurepaire, Jones, Thiering, Saunders \& Tennant (1994) observaram resultados diferentes, relatando que as mulheres, quando comparadas aos homens, apresentam níveis mais elevados de ansiedade e depressão, autoestima mais baixa, culpa e vergonha associadas à infertilidade. A maior dificuldade para ambos é a ansiedade, que está acima dos níveis tidos como normais.

Boivin et al. (1998), realizando avaliação diária de homens e mulheres durante o tratamento em cada estágio da FIV, observaram que a infertilidade e o tratamento têm impacto negativo maior nas mulheres que nos homens.

Após a falha de um primeiro ciclo, homens e mulheres apresentam aumento significativo de ansiedade e sintomas depressivos (Newton, Hearn \& Yuzpe, 1990;
Verhaak et al., 2001). Embora as médias dos escores não sejam clinicamente elevadas e a maioria lide adequadamente, a prevalência de depressão leve e moderada cresce substancialmente, principalmente entre as mulheres.

Recentes estudos têm mostrado relação negativa entre stress e sucesso de FIV (Boivin \& Takefman, 1995; Verhaak et al., 2001). Níveis mais altos de depressão e ansiedade parecem estar relacionados a menores índices de gravidez. A relevância clínica desses achados impele à necessidade de maiores investigações nessa área.

Este estudo propôs avaliar e comparar os estados emocionais de casais com infertilidade primária, quando submetidos ao primeiro ciclo de um tratamento de FIV, e verificar a relação entre estado emocional das mulheres e ocorrência de gravidez.

\section{Método}

\section{Participantes}

Participaram do estudo vinte casais que realizaram o primeiro procedimento de FIV no Setor de Reprodução Humana da Universidade Federal de São Paulo (Unifesp), São Paulo, Brasil. Foram incluídos no estudo casais com infertilidade conjugal primária de causa feminina, com mulheres com até 35 anos de idade. Este estudo foi aprovado pelo Comitê de Ética em Pesquisa da Unifesp (protocolo no 1018/02) e os pacientes assinaram o consentimento informado antes de iniciar o estudo.

\section{Instrumentos e Procedimentos}

Cada membro do casal foi avaliado individualmente pela psicóloga da equipe entre o $5^{\circ}$ e o $10^{\circ} \mathrm{dia}$ 
após a transferência dos pré-embriões. Os seguintes instrumentos foram utilizados:

1) Entrevista estruturada: questões sobre dados sociodemográficos (idade, sexo, escolaridade e atividade profissional) e dados da história clínica da infertilidade (tempo de união, tempo de infertilidade, tempo de diagnóstico e tratamentos anteriores).

2) Questionário de Saúde Geral (GHQ-12) (Goldberg, 1972): instrumento com 12 itens, utilizado para avaliar morbidade psiquiátrica e identificar prováveis casos e prováveis não casos. O escore total varia de 0 a 12; escores $\geq 4$ indicam a presença de sintomas psicoemocionais (prováveis casos). Estudo realizado com a população brasileira indicou boa validade e consistência interna (Mari \& Williams, 1985).

3) Inventário de Depressão de Beck (BDI) (Beck, Ward, Mendelson \& Mock Erbaugh, 1961): consiste de 21 categorias de sintomas e atitudes que descrevem sintomas depressivos. A pontuação varia de 0 a 63; escores mais altos indicam maior severidade de depressão. Foi utilizado o ponto de corte 20 para caracterizar depressão (Kendall, Hollon, Beck, Hammen \& Ingram, 1987). Foram realizados estudos enfocando as propriedades psicométricas da versão em língua portuguesa e os resultados evidenciaram boa validade de construto (Gorenstein, Pompéia \& Andrade, 1995) e de validade discriminante (Gorenstein \& Andrade, 1996).

4) Escala de Depressão de Hamilton (HAM-D) (Hamilton, 1960): instrumento desenvolvido para avaliação e quantificação dos sintomas depressivos. Foi utilizada a versão de 17 itens, cujos escores variam de 0 a 50 pontos; escores mais altos indicam depressão. Foi utilizado o ponto de corte 18 para caracterizar depressão moderada (Del Porto, 1989; R.A. Moreno \& D.H. Moreno, 1998). As informações foram obtidas em entrevista, utilizando-se o manual para entrevista da HAM-D (Williams, 1988). A escala é considerada instrumento válido para avaliar sintomas depressivos no contexto brasileiro (Dratcu, da Costa Ribeiro \& Calil, 1987).

5) Escala de Auto-estima de Rosenberg (Rosemberg, 1965): escala de dez itens que avalia sentimentos relacionados ao próprio indivíduo. Os escores variam de 0 a 30; escores mais altos refletem baixos níveis de autoestima. É considerada adequada ao contexto brasileiro, com bons índices de reprodutibilidade e validade (Dini, 2000).

\section{Análise}

O teste de Wilcoxon foi utilizado para comparar os membros de cada casal em relação aos escores obtidos nas escalas (Siegel \& Castellan, 1988). O teste de McNemar foi usado para estudar as discordâncias entre os componentes do casal para cada um dos itens das escalas utilizadas (Remington \& Schork, 1970). Para comparação dos grupos de mulheres com ou sem sintomas psicoemocionais, em relação à escolaridade, atividade profissional, tratamentos anteriores, etiologia da infertilidade e ocorrência de gravidez, foi utilizado o teste exato de Fisher (Siegel \& Castellan, 1988). O teste de Mann-Whitney (Siegel \& Castellan, 1988) foi utilizado para comparar os grupos de mulheres com ou sem sintomas psicoemocionais, em relação à idade, tempo de união, de infertilidade e de diagnóstico. Em todos os testes $p<0,05$ foi considerado significativo.

\section{Resultados}

A idade média das mulheres foi de 31,4 anos (variação de 24 a 35 anos) e dos homens foi de 34,8 anos (variação de 28 a 43 anos). Todos os homens estavam empregados, enquanto $75 \%$ das mulheres trabalhavam fora de casa, 10\% estavam desempregadas e 15\% exerciam atividades domésticas. 0 nível educacional de homens e mulheres foi similar, 70\% apresentando nível superior. O tempo de união e de infertilidade variou de 2 a 10 anos, com média de 5,3 anos (tempo de união) e de 3,7 anos (tempo de infertilidade). As causas da infertilidade feminina foram: alterações tubárias (65\%), anovulação crônica (10\%), endometriose (5\%) e sem causa aparente (20\%).

Não houve relação significativa entre variáveis sociodemográficas, dados da história clínica e medidas psicológicas.

Comparando-se as respostas dos homens e mulheres nas escalas GHQ-12, Hamilton e BDI, verificou-se que os escores das mulheres foram significativamente maiores que os dos homens. Além disso, a distribuição dos escores da escala de autoestima de Rosenberg concentrou-se em maiores valores para as mulheres (Tabela 1). 
Tabela 1. Comparação das médias dos escores de homens e mulheres nas escalas GHQ-12, HAM-D, BDI e Rosenberg ( $n=20$ casais). Unifesp, São Paulo (SP), 2003.

\begin{tabular}{lcccc}
\hline Escalas & Homens & Mulheres & Wilcoxon & $p$ \\
\hline GHQ-12 & 0,6 & 2,7 & $T=4,0$ & 0,001 \\
HAM-D & 2,1 & 5,6 & 3,43 & 0,003 \\
BDI & 3,2 & 6,3 & 2,03 & 0,021 \\
Rosenberg & 4,5 & 7,7 & 2,62 & 0,004 \\
\hline
\end{tabular}

Teste de Wilcoxon Z crítico = 1,96. GHQ-12: questionário de saúde geral; HAM-D: escala de depressão de Hamilton; BDI: inventário de depressão de Beck

Segundo o GHQ-12, 25\% das mulheres obtiveram escores $\geq 4$, caracterizando presença de sintomas psicoemocionais, enquanto os homens não apresentaram sintomas.

As escalas de depressão evidenciaram diferentes classificações. Na Escala de Depressão de Hamilton, 30\% das mulheres foram classificadas com depressão leve, enquanto, no BDI, 15\% delas obtiveram essa classificação. De acordo com essas duas escalas, os homens não apresentaram sintomas.

As médias dos escores do BDI (6,3 para mulheres e 3,2 para os homens) ficaram abaixo das médias da população normativa $(9,7$ para mulheres e 7,1 para homens) (Gorenstein, Andrade, Vieira Filho, Tung \& Artes,
1999). Os escores das demais escalas não foram comparados com os da população geral porque não há estudos com grupos normativos.

Na distribuição da frequência de respostas positivas dos casais para cada item das escalas utilizadas, identificou-se que as mulheres apresentaram de forma estatisticamente significativa maiores pontuações nos indicadores de ansiedade e sintomas depressivos (Tabela 2).

A gravidez ocorreu em quatro mulheres (20\%), sendo confirmada pela dosagem do hormônio coriônico gonadotrófico e batimento cardíaco fetal à ultrassonografia na sétima semana de gestação. As mulheres que apresentaram sintomas psicoemocionais, segundo o GHQ-12, não engravidaram (Tabela 3).

Tabela 3. Relação entre sintomas psicoemocionais medidos pelo GHQ-12 e ocorrência de gravidez $(n=20)$. Unifesp, São Paulo (SP), 2003.

\begin{tabular}{lccc}
\hline \multirow{2}{*}{ GHQ-12 } & \multicolumn{2}{c}{ Gravidez } & Total \\
\cline { 2 - 3 } & Sim & Não & \\
\hline Com sintomas & 0 & 5 & 5 \\
Sem sintomas & 4 & 11 & 15 \\
\hline Total & 4 & 16 & 20 \\
\hline
\end{tabular}

Teste exato de Fisher $p=0,281$ (ns); GHQ-12: questionário de saúde geral; ns: não significativa.

Tabela 2. Discordâncias significativas entre os componentes dos casais em itens das escalas utilizadas. Unifesp, São Paulo (SP), 2003.

\begin{tabular}{|c|c|c|c|c|c|c|}
\hline & \multirow[b]{2}{*}{ Itens } & \multicolumn{2}{|c|}{ Mulheres* } & \multicolumn{2}{|c|}{ Homens* } & \multirow{2}{*}{$p^{\mathbf{b}}$} \\
\hline & & $\mathrm{n}$ & $\%$ & $\mathrm{n}$ & $\%$ & \\
\hline & \multicolumn{6}{|l|}{ GHQ-12 } \\
\hline & Tensão e nervosismo & 11 & 55 & 5 & 25 & 0,035 \\
\hline & Capacidade de manter atenção & 6 & 30 & 1 & 5 & 0,031 \\
\hline & Tristeza e depressão & 6 & 30 & 0 & 0 & 0,015 \\
\hline & \multicolumn{6}{|l|}{ HAM-D } \\
\hline \multirow{9}{*}{ 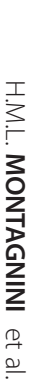 } & Humor depressivo & 8 & 40 & 0 & 0 & 0,039 \\
\hline & Incapacidade ou fadiga para atividades diárias & 5 & 25 & 0 & 0 & 0,031 \\
\hline & Sintomas somáticos & 10 & 50 & 2 & 10 & 0,010 \\
\hline & Redução da libido & 13 & 65 & 5 & 25 & 0,010 \\
\hline & Auto-observação aumentada & 19 & 95 & 1 & 5 & 0 \\
\hline & \multicolumn{6}{|l|}{$B D /$} \\
\hline & Preocupação com a saúde & 12 & 60 & 4 & 20 & 0,019 \\
\hline & Diminuição da libido & 13 & 65 & 4 & 20 & 0,011 \\
\hline & \multicolumn{6}{|l|}{ Rosenberg } \\
\hline 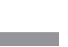 & Sentimento de inutilidade & 5 & 25 & 0 & 0 & 0,031 \\
\hline 478 & \multicolumn{6}{|c|}{$\begin{array}{l}\text { a Número de pacientes que responderam positivamente aos itens; }{ }^{\mathbf{b}} \text { Teste de McNemar }(p<0,05) \text {. GHQ12: questionário de saúde geral; HAM-D: escala } \\
\text { de depressão de Hamilton; BDI: inventário de depressão de Beck. }\end{array}$} \\
\hline
\end{tabular}




\section{Discussão}

Em todas as escalas utilizadas, os escores das mulheres foram significativamente maiores que os dos homens. Vários estudos que compararam o estado emocional de homens e mulheres encontraram resultados semelhantes (Beaurepaire et al., 1994; Beutel et al., 1999, Newton et al., 1990; Slade, Emery \& Lieberman, 1997).

Essas diferenças podem ser atribuídas a vários fatores, entre eles, os níveis de exigência aos quais cada membro do casal é submetido durante o tratamento, que é mais invasivo nas mulheres. Berg, Wilson e Weingartner (1991) concluíram que algumas mulheres apresentaram maior investimento na maternidade, considerando-a algo central para sua identidade. Para elas, a infertilidade passou a ser o maior problema de suas vidas e, ter um filho, o objetivo principal, o que se converteu em fonte de sofrimento psíquico. Outro aspecto que deve ser considerado é que, neste estudo, a causa da infertilidade conjugal é feminina, podendo levar as pacientes a se sentirem mais responsáveis pela ausência de filho e culpadas perante o companheiro.

Este dado pode justificar, em parte, a ausência de sintomas no grupo dos homens. Quando a infertilidade é masculina, a experiência e a resposta emocional dos homens são diferentes, pois têm que lidar com as inconveniências do tratamento e atitude dos outros diante da infertilidade; sentem-se, nessa situação, responsáveis pela dificuldade que o casal vive (Mahstedt, 1985).

Para entender de que maneira se manifestaram as diferenças dos estados emocionais entre homens e mulheres, observaram-se os itens em que as discordâncias se mostraram significantes. Estes se referem ao humor depressivo e à ansiedade, presentes no momento em que o casal aguardava o resultado do tratamento da FIV. Tal estado emocional levou as mulheres a uma maior dificuldade de se vincularem às atividades diárias, apresentando-se mais voltadas para o momento que estavam vivendo, como o tratamento e a expectativa do resultado. Isto poderia justificar a diminuição do interesse sexual das mulheres, porém, como a avaliação foi pontual, não foi possível determinar se a alteração sexual estende-se por um período de tempo maior.
Dezenove mulheres (95\%) apresentaram maior observação do próprio corpo, voltando-se mais para si, monitorando seus corpos em busca de indícios de gravidez. Vale salientar que alguns dos sintomas apresentados, referentes ao aspecto somático da ansiedade e alteração do humor, confundem-se com efeitos colaterais dos medicamentos utilizados pelas mulheres para realizar o procedimento de FIV. Tal aspecto deve ser considerado para analisar os resultados com maior cautela, considerando-se a dificuldade em discriminar quais e quantos desses sintomas foram decorrentes da ansiedade ou dos medicamentos utilizados.

Durante as entrevistas, os homens (40\%) mostraram-se mais agitados e inquietos que as mulheres (20\%), segundo a escala HAM-D. Isso indica maior desconforto e constrangimento dos homens diante da situação de serem avaliados, o que os leva a darem respostas mais aceitas socialmente (Berg et al., 1991). Este dado deve ser considerado para compreender a ausência total de sintomas psicoemocionais no grupo dos homens.

As escalas HAM-D e BDI apresentaram diferenças no modo de pontuação e em suas estruturas, priorizando diferentes categorias de sintomas. A escala HAM-D considera mais os sintomas somáticos (28\%), cognitivos (28\%) e ansiosos (16\%), ao passo que o BDI valoriza mais os sintomas cognitivos (52\%) e somáticos (29\%). Essas diferenças justificam as diferentes classificações que ocorreram. Segundo a HAM-D, 30\% das mulheres apresentaram depressão leve, enquanto no BDI, 15\% das mulheres apresentaram disforia e depressão moderada. Os homens não apresentaram sintomas. Embora as mulheres tenham apresentado escores superiores aos de seus companheiros, os valores não alcançaram, em nenhuma das escalas, níveis que indicassem alterações clínicas relevantes.

Os escores de homens e mulheres inférteis no BDI encontraram-se abaixo dos valores da população normativa, talvez devido ao otimismo dos casais diante da perspectiva de terem o filho desejado, confirmando achados de outros estudos (Beaurepaire et al., 1994; Hearn et al., 1987; Slade et al., 1997; Verhaak et al., 2001).

As diferenças no estado emocional começam a se evidenciar a partir do segundo procedimento, no qual as mulheres apresentam escores de depressão clinicamente mais elevados, quando comparados ao 
primeiro procedimento (Beaurepaire et al., 1994; Beutel et al., 1999). No estudo aqui descrito, as mulheres que apresentaram sintomas psicoemocionais possivelmente apresentaram maior dificuldade em lidar com o tratamento de FIV. Levando-se em consideração a tendência de haver aumento dos índices de depressão nos procedimentos consecutivos, essas mulheres constituem um potencial grupo de risco. Assim, é importante identificar e fornecer auxílio psicológico às pacientes com manifestações sintomáticas leves, para que adquiram meios mais eficazes de lidar com a situação e evitar a exacerbação dos sintomas apresentados.

Apesar de não ter havido relação significativa entre sintomas psicoemocionais e ocorrência de gravidez, nenhuma das mulheres com tais sintomas engravidou, aspecto que poderia ser mais bem avaliado em um grupo maior de pacientes.

A relação entre estado emocional e ocorrência de gravidez remete a situações vividas por algumas mulheres com dificuldade para engravidar e que engravidam naturalmente após adoção, desistência ou interrupção de tratamento para infertilidade, ou mesmo depois de terem um filho por fertilização in vitro (Hennelly, Harrison, Kelly, Jacob \& Barrett, 2000).

Nos estudos realizados para verificar a relação entre aspectos psicológicos e ocorrência de gravidez, os resultados mostram controvérsias, o que pode ser atribuído à utilização de diferentes instrumentos, assim como ao momento da avaliação, considerando-se que há alterações do estado emocional conforme a fase do tratamento, como os efeitos colaterais dos medicamentos.

Este estudo realizou a avaliação após a transferência de pré-embriões, retratando com maior exatidão o estado emocional no período da implantação dos mesmos, no qual a interação entre fatores biológicos e psicológicos tem maior influência na ocorrência da gravidez. Este aspecto deve ser mais estudado pela sua relevância clínica, pois, com a promoção de melhores condições emocionais, pode haver um aumento na taxa de gravidez em tratamento de fertilização assistida.

A infertilidade e seus tratamentos constituem um longo processo, no decorrer do qual emergem diversos sentimentos e comportamentos. Os dados desta pesquisa referem-se a um momento específico dos casais, capturando um processo de ajustamento pontual. Apesar desta limitação, o estudo traz contribuições importantes com implicações práticas para o suporte psicológico dos casais em tratamento de FIV.

\section{Referências}

Beaurepaire, J., Jones, M., Thiering, P., Saunders, D., \& Tennant, C. (1994). Psychosocial adjustment to infertility and its treatment: male and female responses at different stages of IVF/treatment. Journal of Psychosomatic Research, 38 (3), 229-40.

Beck, A. T., Ward, C. H., Mendelson, M., Mock, J., \& Erbaugh, G. (1961). An inventory for measuring depression. Archives of General Psychiatry, 4 (1), 53-63.

Berg, B. J., Wilson, J. F. \& Weingartner, P. J. (1991). Psychological sequelae of infertility treatment: the role of gender and sex-role identification. Social Science and Medicine, 33 (9),1071-1080.

Beutel, M., Kupfer, J., Kirchmeyer, P., Kehde, S., Köhn, F-M., Schroeder-Printzen, I., et al. (1999). Treatment: related stresses and depression in couples undergoing assisted reproductive treatment by IVF or ICSI. Andrologia, 31 (1), 27-35.

Boivin, J., \& Takefman, J. E. (1995). Stress level across stages of in vitro fertilization in subsequently pregnant and non pregnant women. Fertility and Sterility, 64 (4), 802-810.

Boivin, J., Andersson, L., Skoog-Svanberg, A., Hjelmstedt, A., Collins, A., \& Bergh T. (1998). Psychological reactions during in-vitro fertilization: similar response pattern in husbands and wives. Human Reproduction, 13 (11), 3262-7.

Collins, A., Freeman, E. W., Boxer, A. S., \& Tureck, R. (1992). Perceptions of infertility and treatment stress in females as compared with males entering in vitro fertilization treatment. Fertility and Sterility, 57 (2), 350-356.

Del Porto, J. A. (1989). Aspectos gerais das escalas para avaliação de depressão. In Centro de pesquisa em psicobiologia clínica. Escalas de avaliação para monitorização de tratamento com psicofármacos. São Paulo: Escola Paulista de Medicina.

Dini, G. M. (2000). Adaptação cultural, validade e reprodutibilidade da versão brasileira da escala de auto-estima de Rosenberg. Dissertação de mestrado não-publicada, Universidade Federal de São Paulo.

Dratcu, L., Costa Ribeiro, L., \& Calil, H. M. (1987). Depression assesment in Brazil. The first application of the Montgomery-Asberg depression rating scale. The British Journal of Psychiatry, 150, 797-800.

Edelmann, R. J., Connolly K. J., \& Bartlett, H. (1994). Coping strategies and psychological adjustment of couples presenting for IVF. Journal of Psychosomatic Research, 38 (4), 355-364.

Goldberg, D. P. (1972). The detection of psychiatric illness by questionnaire. Maudsley Monograph no 21. London: Oxford University Press. 
Gorenstein, C., \& Andrade, L. (1996). Validation of a portuguese version of the Beck Depression Inventory and the State: Trait Anxiety Inventory in Brazilian subjects. Brazilian Journal of Medical and Biological Research, 29 (4), 453-7.

Gorenstein, C., Andrade, L., Vieira Filho, A. H. G., Tung, T. C., \& Artes, R. (1999). Psychometric properties of the Portuguese version of the Beck Depression Inventory on Brazilian college students. Journal of Clinical Psychology, 55 (5), 553-62.

Gorenstein, C., Pompéia, S., \& Andrade, L. (1995). Scores of Brazilian university students on the Beck depression inventory and the state-trait anxiety inventory. Psychological Reports, 77, 635-641.

Hamilton, M. (1960). A rating scale for depression. Journal of Neurology, Neurosurgery \& Psychiatry, 23, 56-62.

Hearn, M. T., Yuzpe, A. A., Brown, S. E., \& Casper, R. F. (1987). Psychological characteristics of in vitro fertilization participants. American Journal of Obstetrics and Gynecology, 156 (2), 269-74.

Hennelly, B., Harrison, R. F., Kelly, J., Jacob, S., \& Barrett, T. (2000). Spontaneous conceptin after a successful attempt at in vitro fertilization/intracytoplasmic sperm injection. Fertility and Sterility, 73, 774-78.

Kendall, P. C., Hollon, S. D., Beck, A. T., Hammen, C. I., \& Ingram, R. E. (1987). Issues and recommendations regarding use of the Beck Depression Inventory. Cognitive Therapy and Research, 11 (3), 289-99.

Mahlstedt, P. P. (1985). The psychological component of infertility. Fertility and Sterility, 43 (3), 335-45.

Mari, J. J., \& Williams, P. (1985). A comparison of the validity of two psychiatric screening questionnaires (GHQ-12 and SRQ-20) in Brazil, using Relative Operating Characteristic (ROC) analysis. Psychological Medicine, 15, 651-9.

Moreno, R. A., \& Moreno, D.H. (1998). Escalas de depressão de Montgomery \& Asberg (MADRS) e de Hamilton
(HAM-D). Revista de Psiquiatria Clínica, 25 (5, edição especial), 262-272.

Newton, C. R., Hearn, M. T., \& Yuzpe, A. A. (1990). Psychological assessment and follow-up after in vitro fertilization: assessing the impact of failure. Fertility and Sterility, 54 (5), 879-86.

Remington, R. D., \& Schork, M. A. (1970). Statistics with applications to biological and health sciences. Englewood Cliffs, New Jersey: Prentice-Hall.

Rosenberg, M. (1965). Society and the adolescent self image. Princeton: Princeton University Press.

Siegel, S., \& Castellan, J. R. (1988). Nonparametric statistics. 2nd ed. New York: McGraw-Hill.

Slade, P., Emery, J., \& Lieberman, B. A. (1997). A prospective, longitudinal study of emotions and relationships in in-vitro fertilization treatment. Human Reproduction, 12 (1), 183-90.

Thiering, P., Beaurepaire, J., Jones, M., Saunders, D., \&Tennant C. (1993). Mood state as a predictor of treatment outcome after in vitro fertilization/embryo transfer technology (IVF/ET). Journal of Psychosomatic Research, 37 (5), 481-91.

Verhaak, C. M., Smeenk, J. M. J., Eugster, A., van Minnen, A., Kremer, J. A. M., \& Kraaimaat, F. W. (2001). Stress and marital satisfaction among women before and after their first cycle of in vitro fertilization and intracytoplasmic sperm injection. Fertility and Sterility, 76 (3), 525-31.

Williams, J. B. W. (1988). A structured interview guide for the Hamilton depression rating scale. Archives of General Psychiatry, 45 (8), 742-7.

Wright, J., Duchesne, C., Sabourin, S., Bissonnette, F., Benoit, J., \& Girard, Y. (1991). Psychosocial distress and infertility: men and women respond differently. Fertility and Sterility, 55 (1), 100-8.

Recebido em: 13/9/2007

Versão final reapresentada em: 7/4/2008

Aprovado em: 24/4/2008 